\title{
CARDIAC STIMULATION THROUGH INDUCED CURRENTS
}

\author{
OANA DROSU, MARILENA STANCULESCU \\ University Politehnica of Bucharest, Department of Electrical Engineering, Spl. Independentei, no 313 \\ E-mail: oana.drosu@upb.ro
}

\begin{abstract}
This paper presents the magnetic cardiac stimulation, more precisely the stimulation with an electric field produced by a time-variable magnetic field. The modeling within this domain has in view the investigation of the induced currents in the body and the characteristics of the defibrillation coil (shape, number of turns, length) and the applied stimulus.
\end{abstract}

Keywords: magnetic stimulation of the cardiac tissue, numerical modeling, induced currents.

\section{INTRODUCTION}

The purpose of this work is to investigate the electric field distribution and the induced currents in a section of the thorax through the time variation of a magnetic field produced by an inductor (coil) surrounding the patient' thorax [1].

It has been investigated to what extend these currents produce defibrillation, applying the criterion of exceeding the value of the excitability threshold for approximately $75 \%$ of the myocardium [2].

The characteristics of the induced currents are determined by the parameters of the exterior circuit, the defibrillation coil being a part of this circuit.

The values corresponding to the components of external series circuit: R, L, C will be chosen and the problem will be solved, determining the space distribution and the time variation of the induced currents.

Based on the obtained results, the characteristics of the defibrillation coil can be estimated, more precisely the ratio between the coil turn number and its length will be determined.

\section{PROBLEM FORMULATION}

\section{A. Physiscal and mathematical model}

The electromagnetic field state is a dynamic one and the anatomical structure has predominantly resistive electric properties.

Therefore, the adopted model is that of a linear, piecewise homogenous conductor from the electric point of view, subjected to electromagnetic field diffusion.

The model of massive conductor placed inside an infinite long solenoid has been chosen. The outer circuit consists of a DC source that powers the capacitor C. This discharges on a RL series circuit (taking into account the patient's resistance), producing the excitation current in the defibrillation coil.

For the outer circuit the values has been taken from the literature [3] $(\mathrm{R}=54.5 \mathrm{~m} \Omega, \mathrm{L}=110 \mu \mathrm{H}, \mathrm{C}=4 \mathrm{mF}, \mathrm{U}=950$ $\mathrm{V})$. This values can be modified later to optimize the cardiac defibrillation using magnetic stimulation.

\section{B. Domain and material properties}

The domain has a plane-parallel geometry, represented by a transversal crossection through the assembly consisting of the coil and the thorax of the patient.

The section through the thorax has been divided in eight sub-domains: thorax wall, muscles, bones (ribs and spine), lungs, interstitial fluid, myocardium, atria and ventricles filled with blood, esophagus; the values for the corresponding conductivities have been taken from literature [2].

All these media have been considered to be linear, isotropic (including also the intercostal muscles for which an average conductivity value has been chosen) and homogenous.

The dielectric and magnetic properties of the structure are those corresponding to the free space: therefore, the electric permittivity will be $\varepsilon=\varepsilon_{0}$, respectively the magnetic permeability $\mu=\mu_{0}[2]$, [4].

\section{Electromagnetic field equations}

The general and the material laws, corresponding to the electromagnetic field problem, are [5] :

- local form of the magnetic circuit law:

$$
\operatorname{rot} \vec{H}=\vec{J}
$$

- local form of the electromagnetic induction law:

$$
\operatorname{rot} \vec{E}=-\frac{\partial \vec{B}}{\partial t}
$$

- local form of the magnetic flux law

$$
\operatorname{div} \vec{B}=0
$$

- local form of the electric conduction law (in homogenous conditions): 


$$
\vec{J}=\sigma \vec{E}
$$

- connection law in magnetic field:

$$
\vec{B}=\mu \vec{H}
$$

The defibrillation coil, of circular shape, represents the boundary of the domain, where the field sources are given in the form of the condition:

$$
\vec{H}_{t}=\vec{f}
$$

The initial condition is associated to the quantity to be derived, i.e. $\vec{B}$.

At $\mathrm{t}=0$, the value of $\vec{B}(0)$ is given. It is known that $\operatorname{div} \vec{B}(0)=0$ and it has been chosen $\vec{B}(0)=0$, so it results that:

$$
\vec{H}(0)=0
$$

The uniqueness of the solution is provided by the equations $1-7$.

In transient state, according to the above equations, the field quantities satisfy a diffusion type equation, written in terms of the magnetic field strength:

$$
\operatorname{rot} \operatorname{rot} \vec{H}=-\mu \sigma \frac{\partial \vec{H}}{\partial t},
$$

or, taking into consideration the magnetic flux law:

$$
\Delta \vec{H}=\mu \sigma \frac{\partial \vec{H}}{\partial t}
$$

Taking into account that the studied problem is bidimensional, then the magnetic field diffusion equation can be written in particular scalar form as follows:

$$
\frac{\partial^{2} H}{\partial x^{2}}+\frac{\partial^{2} H}{\partial y^{2}}=\mu \sigma \frac{\partial H}{\partial t}
$$

Where: $H=H(\mathrm{x}, \mathrm{y})$

\section{Boundary conditions}

On the boundary, represented by the circular coil contour, a Dirichlet type condition has been imposed, giving $H$ along the boundary.

From the magnetic field law, on the discontinuity surfaces, considering the separation surface between the outer and the inner part of the coil, it results:

So

$$
\operatorname{rot}_{s} H=J_{s}
$$

$$
H_{t \text { int }}-H_{t e x t}=J_{s}
$$

Because $H_{\text {text }}=0$, it results:

$$
H_{f r}=H_{t \text { int }}=J_{s}=\frac{N i}{l}
$$

where:

- $\quad N=$ the number of turns of the coil;

- $\quad i=$ the current through the coil;

- $l=$ the length of the coil.

- As previously shown, the initial condition is chosen to be $H(0)=0$.

\section{E. Diffusion equation scaling}

Because the scaled forms (dimensionless) are more appropriate for the numerical models [5], the following reference scalar quantities have been introduced:

- $H_{0}(\mathrm{~A} / \mathrm{m})$ - the reference magnetic field strength (the maximum value of the impulse will be chosen);

- $\quad L_{0}(\mathrm{~m})$ - the reference length (chosen as the average length of the field line measured at the periphery of the thorax, for the electric stimulation with two electrodes placed on antero-posterior position);

- $\quad \sigma_{0}(\mathrm{~S} / \mathrm{m})$ - the reference electric conductivity [6] (the equivalent conductivity of the structure).

From the electromagnetic field laws, it results:

$$
B_{0}=\mu H_{0}
$$

(according to the constitutive law of magnetic field);

$$
J_{0}=H_{0} / L_{0}
$$

(according to magnetic circuit law);

$$
E_{0}=J_{0} / \sigma_{0}=H_{0} /\left(\sigma_{0} L_{0}\right)
$$

(according to conduction law).

The scaled field quantities are:

$$
\widetilde{B}=\frac{B}{B_{0}}, \widetilde{H}=\frac{H}{H_{0}}, \widetilde{J}=\frac{J}{J_{0}}, \widetilde{E}=\frac{E}{E_{0}} .
$$

By scaling, the following equation results:

$$
\frac{1}{\mu \sigma_{0} L_{0}{ }^{2}} \frac{\sigma_{0}}{\sigma}\left(\frac{\partial^{2} \tilde{H}}{\partial \tilde{x}^{2}}+\frac{\partial^{2} \tilde{H}}{\partial \tilde{y}^{2}}\right)=\frac{1}{t_{0}} \frac{\partial \tilde{H}}{\partial \tilde{t}}
$$

Where: $\tilde{x}=\frac{x}{L_{0}} ; \quad \tilde{y}=\frac{y}{L_{0}} ; \quad \tilde{t}=\frac{t}{t_{0}}$.

For time scaling, by choosing the value: 


$$
t_{0}=\mu \sigma_{0} L \underset{0}{2}
$$

and denoting by:

$$
\sigma_{e}=\sigma / \sigma_{0}
$$

the scaled diffusion equation will be written as:

$$
\frac{1}{\sigma_{e}}\left(\frac{\partial^{2} \widetilde{H}}{\partial \widetilde{x}^{2}}+\frac{\partial^{2} \widetilde{H}}{\partial \widetilde{y}^{2}}\right)=\frac{\partial \widetilde{H}}{\partial \widetilde{t}}
$$

By comparison with the non-scaled equation, the following equivalences result:

$$
\mu \leftrightarrow 1 \text { and } \sigma \leftrightarrow \sigma_{e}
$$

The equation is completed by the scaled boundary condition:

$$
\widetilde{H}_{f r}=\frac{H_{f r}}{H_{0}} .
$$

\section{F. The excitation form}

The efficiency of the applied stimulus depends on many factors, among which there are: the shape, the amplitude and the duration of the impulse.

In this study, the following type of the impulse has been studied: aperiodic damped oscillation (resulting from discharging a capacitor on an RL series circuit).

The shapes of the current waves from the RLC series circuit are given by the following expression:

$$
I_{a m}=U \gamma \sin (\beta t) \exp (-\alpha t) \text {. }
$$

where:

$\mathrm{U}=950 \mathrm{~V}-$ is the capacitor charging voltage and $\alpha, \beta, \gamma$ are:

$$
\begin{gathered}
\alpha=\frac{R}{2 L} . \\
\beta=\sqrt{\frac{1}{L C}-\left(\frac{R}{2 L}\right)^{2}} . \\
\gamma=C \frac{\alpha^{2}+\beta^{2}}{\beta} .
\end{gathered}
$$

The computed values of these parameters are: $\alpha=247.72$, $\beta=1487.06, \gamma=6.113$.

From the relations (13) and then (22) it results:

$$
\widetilde{H}_{f r-a m}=\sin \left(\beta t_{0} \widetilde{t}\right) \exp \left(-\alpha t_{0} \widetilde{t}\right) .
$$

\section{THE NUMERICAL MODEL}

The problem has been numerically solved by using the FEM software MEGA.

The mesh is a mixed one, having both triangular and quadrilateral-elements. The nodal unknown associated to the mesh is the magnetic field strength $\mathrm{H}$, its value within an element being an approximated function of its corresponding values from the element nodes.

The mesh contains 3120 nodes and 3268 elements (Figure 1 ), the corresponding problem being solved using a working station in about two minutes, for a time network of 100 steps.

Since the problem is a diffusion one, a time discretization network has been generated; at each point in time, the software solves a steady state equations system.

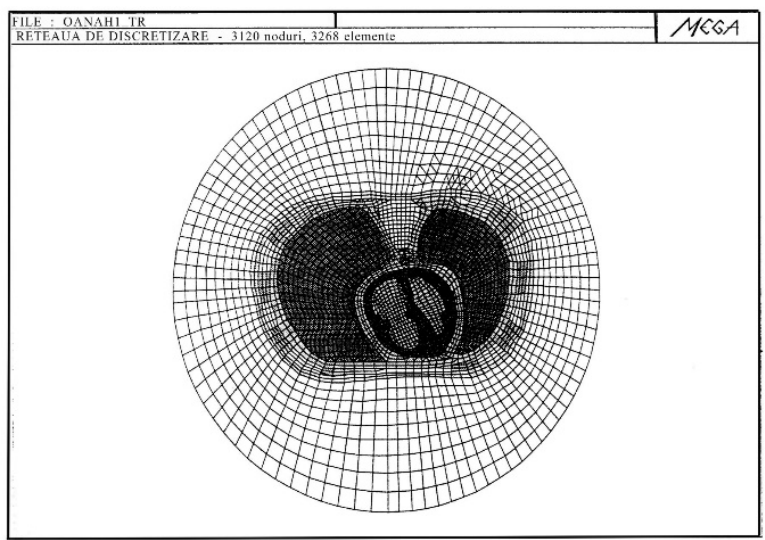

Figure 1. The discretization mesh of the 2D model

For the time network, an accuracy test took place, choosing 100 steps.

The boundary conditions are Dirichlet type conditions, the time variable $\boldsymbol{H}$ being given in the mesh nodes placed on the circle represented by the defibrillation coil.

For the scaled model, there are several steps:

- obtaining the equivalent conductivity, DC, corresponding to the structure;

- considering the magnetic permeability 1;

- scaling the coordinates $\mathrm{x}, \mathrm{y}$;

- computing $\mathrm{t}_{0}$.

By introducing the above-mentioned quantities as input data, practically the scaled equation is solved.

To determine the real values of the problem solution, the obtained results will be multiplied by the considered reference values. The same algorithm has been applied for accuracy test performed for the time network, as shown in Table 1. 
Table 1. Accuracy test for the time network - damped oscillating impulse

\begin{tabular}{|c|l|c|c|c|}
\hline $\begin{array}{c}\text { Time } \\
\text { moment }\end{array}$ & $\begin{array}{c}\text { No. of } \\
\text { steps }\end{array}$ & $\mathbf{J}_{\text {apex }}$ & $\mathbf{J}_{\text {thorax }}$ & $\begin{array}{c}\mathbf{J}_{\text {heart }} \\
\text { integral }\end{array}$ \\
\hline \multirow{3}{*}{$10^{-3}$} & 50 & 2.91301 & 4.86751 & $0.55293 \times 10^{-2}$ \\
\cline { 2 - 5 } & 100 & 2.84292 & 4.37987 & $0.49419 \times 10^{-2}$ \\
\cline { 2 - 5 } & 200 & 2.81009 & 4.20195 & $0.47092 \times 10^{-2}$ \\
\hline \multirow{3}{*}{$10^{-2}$} & 50 & 0.32029 & 0.22713 & $0.14981 \times 10^{-2}$ \\
\cline { 2 - 5 } & 100 & 0.39958 & 0.45827 & $0.16402 \times 10^{-2}$ \\
\cline { 2 - 5 } & 200 & 0.41993 & 0.47639 & $0.17096 \times 10^{-2}$ \\
\hline
\end{tabular}

The 100 steps option has been chosen for a minimal computational effort, without affecting the solution.

The Figures 2 and 3 present the magnetic field strength and the current density spectrum for different time moments.

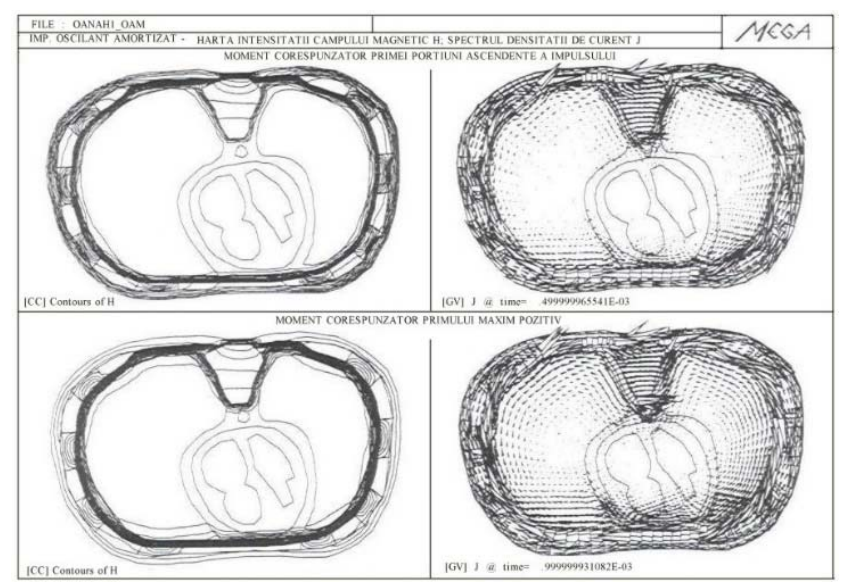

Figure 2. Magnetic field strength and current density for the first increasing part of the impulse

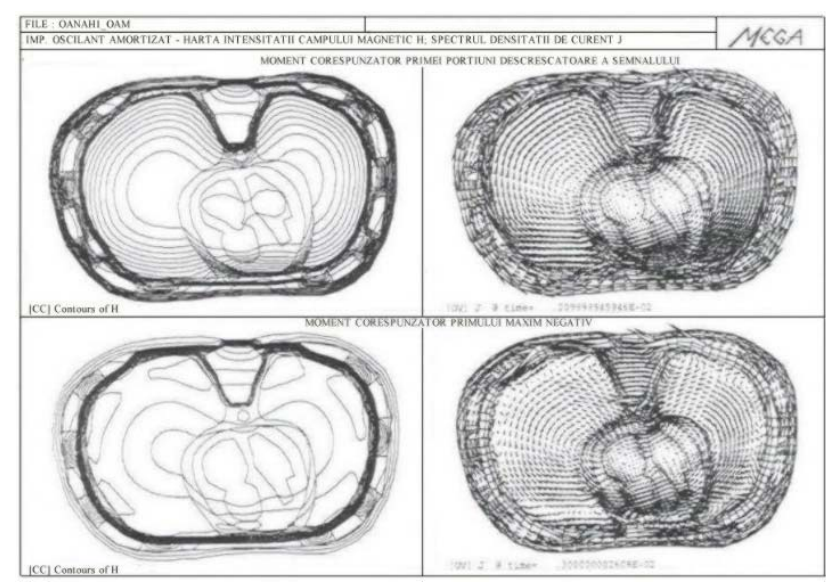

Figure 3. The magnetic field strength and current density for the first decreasing part of the impulse

By analyzing the current density corresponding to four chosen moments, one can notice the change of the induced currents direction during the first oscillation period.

The Figure 4 presents the time variation of the induced currents in different regions of the thorax, apex and interstitium.

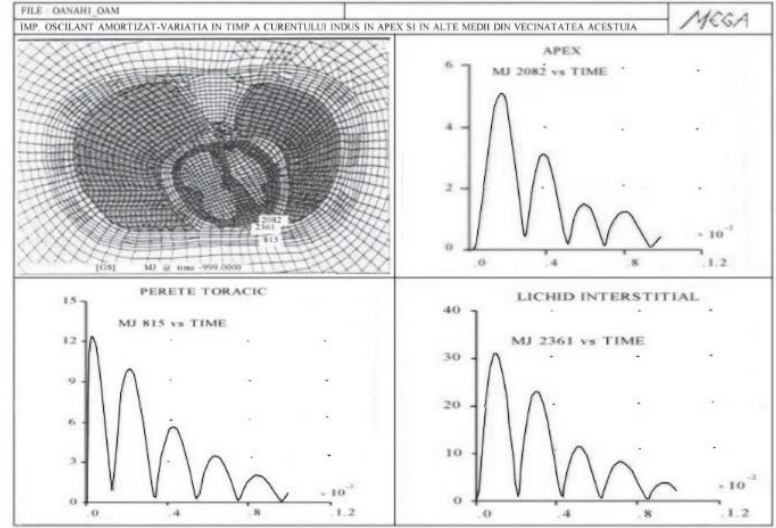

Figure 4. The induced currents in the apex, thorax and interstitium

We conclude that the maximum values are obtained for the interstitial fluid while the minimum ones are characteristic to the bone tissues.

Drawing a curve through the superior points (positive peaks) of the graph of the induced current in the heart apex, one can see a similarity with the shape of the impulse variation applied to the electrodes in the case of electric stimulation [7], the impulse of the type:

$$
I=\frac{I_{e x}}{1-\exp \left(-\frac{T}{\tau}\right)}
$$

where:

$\mathrm{I}_{\mathrm{ex}}=$ excitation threshold, independent of tissue;

$\mathrm{T}=$ effective duration of the excitation;

$\tau=$ time constant, tissue dependent.

The first maximum moment for the induced current is $\widetilde{t}$ $=1.7 \times 10^{-3} \mathrm{~s}$. Considering $\mathrm{t}_{0}=4 \times 10^{-2}$, it results $t=6.8 \times 10^{-}$ ${ }^{5} \mathrm{~s}$.

For an impulse of $\mathrm{B}_{0}=0.1 \mathrm{mT}$, respectively $\mathrm{H}_{0}=79.617$ $\mathrm{A} / \mathrm{m}$, the reference value is computed for the other quantities.

The maximum value of the current density from the heart apex is $\widetilde{J}_{\max }=5.04$.

From $J_{\max }=J_{0} \widetilde{J}_{\max }$, for $\mathrm{J}_{0}=128.378 \mathrm{~A} / \mathrm{m}^{2}$, the following value is obtained:

$$
J_{\max }=647.471 \mathrm{~A} / \mathrm{m}^{2}>250 \mathrm{~A} / \mathrm{m}^{2}=J_{\text {threshold }}
$$

The obtained value is higher than the threshold one ( $J_{\text {max }} \cong 2.5 x J_{\text {prag }}$ ) and at the same time it does not exceed the value corresponding to the occurrence of unwanted side-effects.

At this value, approximately $70 \%$ of the myocardium mass is subjected to defibrillation. 
The limits can be established by enclosing the maximum value of the current density between the threshold values:

and

$$
\mathrm{J}_{\text {threshold }}=250 \mathrm{~A} / \mathrm{m}^{2}
$$

obtaining for $\mathrm{J}_{0}$ :

$$
4 \mathrm{xJ}_{\text {threshold }}=1000 \mathrm{~A} / \mathrm{m}^{2},
$$

$$
49.603 \leq J_{0}=\frac{J_{\max }}{\widetilde{J}_{\max }} \leq 198.41 \mathrm{~A} / \mathrm{m}^{2}
$$

and

and

$$
30.75 \leq H_{0}=J_{0} L_{0} \leq 123.03 \mathrm{~A} / \mathrm{m}
$$

$$
0.038 m T \leq B_{0} \leq 0.154 m T
$$

\section{CONCLUSIONS}

Analyzing the obtained results (synthesized in Table 2), one can notice that, as a consequence of the used impulse, for a good design of the external circuit the defibrillation takes place right from the first moments in the lower areas of the ventricles, rapidly expanding in the other areas of the myocardium.

Table. 2 Results synthesis

\begin{tabular}{|c|c|c|c|c|}
\hline $\begin{array}{c}\mathbf{J}_{\text {max-apex }} \\
{\left[\mathbf{A} / \mathbf{m}^{2}\right]}\end{array}$ & $\begin{array}{c}\mathbf{J}_{\text {max-thor }} \\
{\left[\mathbf{A} / \mathbf{m}^{2}\right]}\end{array}$ & $\begin{array}{c}\mathbf{J}_{\text {max-thor }} / \\
\mathbf{J}_{\text {max-apex }}\end{array}$ & $\begin{array}{c}\mathbf{J}_{\text {max-apex }} / \\
\mathbf{J}_{\text {thershold }}\end{array}$ & $\begin{array}{c}\text { Stimulated } \\
\text { Mass \% }\end{array}$ \\
\hline 647.47 & 1604.72 & 2.47 & 2.58 & $70 \%$ \\
\hline
\end{tabular}

For a damped oscillating impulse, the first maximum of the current induced in the apex is twice the threshold value.

Also, the myocardium mass sufficiently stimulated by the defibrillation current is $70 \%$ for this excitation type.

Taking into account the percentage of $75 \%$ established for the critical mass by the [1], [3], [6], for a successful defibrillation it is obvious that the damped oscillating impulse is getting considerably close to the validation of this criterion.

On the other hand, a change of the induced current direction is produced, as a consequence of the time variation of its shape.
A possible disadvantage of this type of impulse might be the re-entering in the fibrillation state due to the sufficiently high values of the other maxima or of the first negative one. To avoid this situation, a rapid attenuation of the impulse is suggested, such that the other maximum values not to exceed the threshold value.

\section{ACKNOWLEDGMENTS}

This work has been funded by University Politehnica of Bucharest, through the "Excellence Research Grants" Program, UPB - GEX. Identifier: UPB-EXCELENȚA 2016, Research project title "Stimuli electromagnetici pentru tesuturile vii" (Electromagnetic Stimuli for alive tissues) Contract number 73.

\section{REFERENCES}

[1] O. Drosu, M. Sanculescu, Review on Electromagnetic Stimulation of Alive Tissues, 4 pg., BDI, DOI:10.1515/sbeef-2016-0014), Jan 2016 (Copernicus).

[2] Drosu O., Electromagnetic field in biomedical engineering (Câmpul electromagnetic în bioingineria medicală), Ed. Electra, Bucureşti 2004-ISBN 9737728-03-3.

[3] Yamaguchi M., Andoh T., Goto T., Heart Stimulation by Time-varying Magnetic Fields, Jpn. J. Apl. Physics, vol. 31, pp. 2310-2316, 1992.

[4] Mihaela Morega, Bioelectromagnetism - curs, Ed. Matrix Rom, Bucureşti, 1999.

[5] Mocanu C., Electrical Engineering Electromagnetic field theory (Bazele electrotehnicii Teoria campului electromagnetic), E.D.P., Bucuresti, 1991.

[6] Morega Al. M., Mocanu D., Morega M., Stefan A. A dynamic model for the extracutaneous defibrillation, 75th Anniversary Session of the School of Electrotechnics, 6-7 Dec 1996, p. 97-203.

[7] O. Drosu, A Numerical Model of Internal Electrical Stimulation of the Heart, Proceedings of ATEE Conference-Section 2- CAD in Electrical Engineering, pg. 33-36, ISBN 973-7728-31-9, Bucharest, Romania, Nov. 2004. 